\title{
APRENDIZAGEM BASEADA EM PROBLEMAS E ENSINO EXPOSITIVO: UM ESTUDO COMPARATIVO
}

\author{
PROBLEM-BASED LEARNING AND LECTURE-BASED LEARNING: A COMPARATIVE STUDY
}

\author{
Sara Moutinho, Joana Torres, Clara Vasconcelos* \\ Universidade do Porto, Porto, Portugal \\ Faculdade de Ciências - Centro de Geologia - Unidade de Ensino das Ciências \\ E-mail: sara.moutinho@fc.up.pt, joana.torres@fc.up.pt,csvascon@fc.up.pt
}

\begin{abstract}
Resumo
Este trabalho teve como objetivo comparar a $A B P$ e o ensino expositivo relativamente à construção e retenção do conhecimento. $O$ estudo seguiu uma metodologia de investigação quasiexperimental com uma seleção não-aleatória de participantes. Foi sido aplicado um teste cognitivo em duas fases diferentes, a fase I após o término do programa de intervenção e, outra, a fase II, três meses depois. Os resultados mostram que os alunos do grupo experimental melhoram a retenção do conhecimento da fase I para a fase II, algo que não sucedeu com o grupo de controlo. Este estudo permitiu demonstrar que a introdução da ABP no ensino de Ciências Naturais não afeta negativamente o sucesso escolar e, em comparação com o ensino expositivo, promove uma maior retenção do conhecimento.
\end{abstract}

Palavras-chave: aprendizagem baseada em problemas, ensino expositivo, metodologia de ensino, estudo quasi-experimental, ciências naturais.

\begin{abstract}
The study followed a quasi-experimental research methodology with a non-random selection of participants. The study was divided into two phases, one applied after the intervention program (phase I) and another one applied three months later (phase II). Results show that, concerning the retention of knowledge, students for the experimental group showed improvements of the results of the comparison between phase I and phase II. It was possible to demonstrate that the implementation of PBL in Natural Sciences teaching does not affect negatively educational achievement, and promotes a better retention of knowledge, compared to the PBL.
\end{abstract}

Keywords: problem-based learning, lecture-based learning, teaching methodology, quasi-experimental study, natural sciences. 


\section{INTRODUÇÃO}

A introdução de novas metodologias de ensino em sala de aula sempre foi uma questão controversa (CARRIÓ et al., 2011), principalmente devido ao medo inevitável de fracassar. A preocupação com a extensão dos programas curriculares e a preparação dos alunos para os exames nacionais, muitas vezes sobrepõe-se a qualquer tentativa de melhorar a qualidade do ensino, alterando profundamente esse processo. O desenvolvimento de competências é muitas vezes sobreposto pela aquisição do conhecimento, a fim de garantir resultados escolares satisfatórios em exames padronizados. No entanto, a falta de desenvolvimento de competências revela-se a longo prazo, nomeadamente através da incapacidade de mobilizar conhecimento e aplicá-lo a novas situações do quotidiano. Como tal, garantir o sucesso escolar em disciplinas de Ciências Naturais vai muito além dos resultados quantitativos obtidos em testes nacionais. É fundamental alertar para a necessidade de aprendizagem de outras competências, por exemplo, o raciocínio científico, a auto regulação e a autonomia no processo de aprendizagem (VASCONCELOS e ALMEIDA, 2012). Pelo exposto, cada vez mais se defende a adoção de novas abordagens educacionais visando proporcionar aos alunos o desenvolvimento de competências gerais. Um exemplo de metodologia a destacar é a aprendizagem baseada em problemas ( $A B P$ ), que pretende aumentar as competências de resolução de problemas, a aprendizagem independente e a capacidade de desenvolver trabalho em grupo (PRINCE et al. , 2005).

Os dados aqui apresentados foram obtidos com base numa amostra de 115 alunos, pertencentes a duas escolas do 30 ciclo do ensino básico do Norte de Portugal, e decorre de um projeto que investiga a contribuição da ABP no ensino das ciências para a promoção da cidadania. O principal objetivo deste estudo foi averiguar se a metodologia ABP poderia garantir ganhos cognitivos, e uma maior retenção do conhecimento a longo prazo, em alunos de Ciências Naturais, especialmente quando comparado com o ensino expositivo. Em última análise, o estudo teve como objetivo verificar se a ABP foi pelo menos tão eficaz como o ensino expositivo, no que respeita ao auxílio dos alunos para alcançar o conhecimento necessário para cumprir os objetivos de aprendizagem descritos no currículo nacional de Ciências Naturais. Para atingir os objetivos anteriormente descritos foi desenvolvido e implementado um programa de intervenção (PI), bem como aplicados vários testes cognitivos em momentos diferentes da intervenção. Um primeiro momento foi o da aplicação do pré-teste antes do início da administração do PI. Os pós-testes foram aplicados em duas fases distintas, uma após o término do programa de intervenção (fase I) 
e outra três meses depois (fase II). Após a recolha, os dados foram analisados estatisticamente tendo-se aplicado dois testes estatísticos não paramétricos para a obtenção de resultados significativos.

\section{ENQUADRAMENTO TEÓRICO}

\subsection{Aprendizagem Baseada em Problemas (ABP)}

A aprendizagem baseada em problemas encontra-se inserida numa perspectiva orientada para a investigação, habitualmente designada por Inquiry-Based Learning, porque solicita a curiosidade do aluno para resolver problemas nem sempre com uma única possibilidade de resposta (VASCONCELOS et al., 2012). Além disso, esta metodologia posiciona o questionamento e a investigação no centro do desenvolvimento do processo de aprendizagem. Além disso, uma abordagem investigativa também envolve atividades onde os alunos desenvolvem 0 conhecimento e a compreensão de saberes científicos, bem como espelham os processos segundo os quais os cientistas estudam o mundo natural (NRC, 2008).Por estar enquadrada numa abordagem investigativa, esta metodologia constitui um processo dinâmico, em que a aprendizagem é um processo de pensamento contínuo que envolve flexibilidade e julgamento. Estes processos promovem o desenvolvimento do pensamento crítico e reflexivo sobre o processo em si, envolvendo também aspetos emocionais, como a curiosidade (SADEH e ZION, 2009). Defende-se, assim, que os alunos devem ter a oportunidade de reunir evidências, decidir sobre o seu valor e elaborar explicações científicas coerentes com base nessas evidências recolhidas (KIM, TAN e TALAUE, 2013). Durante as aulas de ciências, onde são implementadas estratégias potenciadoras de investigação, os alunos desenvolvem o seu conhecimento científico, bem como capacidades investigativas relevantes, tais como a identificação de problemas, formulação de questões de investigação, concessão e realização de experiências, comunicação e argumentação de hipóteses, modelos e explicações (ABD-EL-KHALICK et al., 2004; SADEH e ZION, 2009).

Enquanto metodologia de ensino, a ABP centra-se no aluno, incentivando-o a participar ativamente na resolução de problemas do mundo real. A resolução desses problemas é considerada pessoal, social e ambientalmente importante. Além disso, estimula também os alunos a confrontarem o que eles já sabem com o que vão encontrar durante a investigação (GALLAGHER 
et al., 1995; YOON, TREAGUST e CHANDRADRASEGARAN, 2014).Em síntese, é uma metodologia que situa a aprendizagem num contexto de resolução de problemas. Ao permitir ainda que os alunos considerem factos com que são confrontados com a apresentação do cenário problemático, ajuda-os a tornarem-se reflexivos e a desenvolverem o questionamento (VASCONCELOS et al., 2012). Pelo exposto, compreende-se que os elementos principais desta metodologia são o problema (cenário problemático), o grupo e o tutor. O problema leva os alunos a identificarem os objetivos de aprendizagem para a elaboração de uma hipótese. A investigação, a discussão em grupo e a construção de novos conhecimentos leva-os a responderem às perguntas, as quais thes permitirão resolver o problema (CARRIÓ et al., 2011), sempre com a medicação de um professor-tutor.

Segundo Yoon, Treagust e Chandradrasegaran (2014), a aprendizagem suportada numa metodologia ABP poder ser construída individualmente ou em grupos, fomentando-se neste caso as discussões e partilha de opiniões colaborativamente, investigação, reflexão e apresentação do produto final. O trabalho colaborativo dos alunos em pequenos grupos melhora a construção do conhecimento e desenvolve diferentes competências (WONG e DAY, 2008). Apesar da implementação do trabalho colaborativo não ser fácil, a metodologia também apresenta outras potencialidades, uma vez que permite que os alunos partilhem pontos de vista, e os professores orientem melhor o desenvolvimento das diferentes tarefas.

Os problemas mais ou menos bem estruturados (cenários problemáticos) atuam como um estímulo para os processos de aprendizagem dos alunos (WONG e DAY, 2008). Estes cenários ABP devem motivar os alunos a levantar questões e a procurar soluções, recorrendo para isso a atividades de recolha de informação. Os professores que lecionam segundo a metodologia ABP devem procurar criar bons problemas para serem discutidos nas aulas, tendo por base os objetivos claros da aprendizagem. Este processo é de extrema importância pois é através desse problema, que integrará o cenário de problematização, que o professor promove a aprendizagem dos principais conceitos, fatos e processos relacionados com o conteúdo da temática a ser lecionada. Allen, Donham e Bernhardt (2011) explicam que os problemas devem ser cuidadosamente construídos, não só para apresentar aos alunos as questões e dilemas que são importantes para eles, mas também para promover o desenvolvimento de quadros conceituais. Estes problemas 
colocam intencionalmente desafios cognitivos, não fornecendo todas as informações necessárias, motivando, assim, a busca de informação autodirigida para obtenção de explicações.

Depois de desenvolvido o problema e criado um cenário de problematização convidativo (TORRES, PRETO e VASCONCELOS, 2013), o papel do professor passa por fomentar nos alunos a investigação, ajudando-os para que a aprendizagem se torne autodirigida (BARREL, 2007). Embora o processo de ABP conduza por si só a uma aprendizagem mais autónoma por parte dos alunos, os professores devem orientá-los através da promoção de discussões guiadas e moderadas por si, formulação de perguntas que desafiem os alunos a ir mais longe e estimulando a sua participação (ALLEN, DONHAM e BERNHARDT, 2011). Uma das barreiras que surge no uso da ABP é a falta de professores qualificados para desempenhar o papel de facilitadores e mediarem todo o processo de aprendizagem (HMELO-SILVER, 2004). Além disso, as avaliações da maioria dos alunos não contemplam o trabalho em grupo ou a colaboração desenvolvida com a ABP (SAVIN-BADEN, 2004), considerando-se apenas as questões mais conceituais.

Com a introdução da $A B P$ no ensino não universitário (básico e secundário), os professores tiveram necessidade de modificar a metodologia para acomodar grandes turmas, uma maior diversidade dos alunos, problemas de tempo e planificação das aulas, a formação de vários grupos e a falta de adequação do espaço da sala de aula (ALLEN, DUCH e GROH, 1996). Esta metodologia requer uma mudança no paradigma educacional. Como os alunos se tornam construtores ativos do seu conhecimento o papel do professor altera-se de transmissor de informação para facilitador de um processo de resolução de problemas (ALLEN, DONHAM e BERNHARDT, 2011).

Os alunos sujeitos à metodologia $A B P$ são capazes de realizarem uma aprendizagem autónoma, especialmente na definição dos seus objetivos, no planeamento da sua aprendizagem, no acesso e seleção dos seus próprios recursos de aprendizagem, estudando ativamente os materiais e integrando novos conhecimentos na resolução de problemas (YOON, TREAGUST e CHANDRADRASEGARAN, 2014). Por outras palavras, os alunos têm de ser autónomos, assumir a responsabilidade pela sua própria aprendizagem (MACKINNON, 1999), ser solucionadores de problemas ativos e parceiros de cooperação com o outro. Além disso, devem desenvolver a capacidade de coordenar ações e pessoas, atingir metas e monitorizar a sua própria compreensão (PARIS e PARIS, 2001). 


\subsection{Aprendizagem Baseada em Problemas nas aulas de ciências}

A $A B P$ é reconhecida como uma metodologia que pode desenvolver nos alunos o raciocínio científico e auxiliar a aprendizagem de alguns aspetos essenciais da investigação científica, nomeadamente a recolha de factos, a procura de evidências, a procura de soluções, a argumentação e a comunicação dos resultados investigados (VASCONCELOS e ALMEIDA, 2012). Além disso, promove também a compreensão da própria natureza da ciência. A metodologia ABP pode ser efetivamente aplicada no campo das ciências, garantindo a inclusão de alguns componentes dos processos científicos. Gallagher e colaboradores (1995) sugeriu que quatro dos elementos essenciais da ABP podem ser alinhados com a prática da ciência:

i) os problemas devem concentrar-se em conceitos científicos significativos;

ii) deve haver oportunidade para testar as ideias dos alunos através da experimentação ou do trabalho de campo;

iii) os alunos devem gerir os seus próprios dados e

iv) a apresentação das soluções obtidas é fundamental (YOON, TREAGUST e CHANDRADRASEGARAN, 2014).

Esta metodologia tem como objetivo desenvolver competências de comunicação, pensamento crítico, raciocínio e conhecimento científico, tomada de decisão, auto e heteroavaliação. Estas competências são consideradas essenciais para um processo de aprendizagem ao longo da vida (VASCONCELOS, 2012).

No entanto, sempre que uma nova metodologia de ensino é implementada dentro das salas de aula de ciências, muitas dúvidas e críticas surgem por parte dos diretores das escolas, alguns professores, alunos e até mesmo os pais. Vários estudos já forneceram algumas evidências experimentais que apoiam a hipótese de que a metodologia ABP não afeta a aquisição de conhecimento factual dos alunos, quando comparado com um ensino mais tradicional (CARRIÓ et al., 2011).Outros estudos sugerem que a ABP é significativa para o desenvolvimento de competências genéricas e científicas já que os alunos são confrontados com problemas complexos e têm de procurar soluções de forma criativa (CARRIÓ et al., 2011), e também porque a ABP desenvolve competências que muitas vezes são ignoradas no interior das salas de aula de ciências (HMELO-SILVER, 2004; WONG e DAY, 2008). Mesmo na educação em medicina, onde a ABP tem 
sido largamente aplicada e investigada, ainda é necessária uma investigação aprofundada para enriquecer a nossa compreensão sobre a natureza desta metodologia emergente (DOLMANS et al., 2005).

Neste quadro, as nossas alegações seguem a maioria dos estudos relacionados com este método de ensino, que pretendem compreender como é que os alunos aprendem através da metodologia ABP. Além da resenha teórica e das advertências previamente apresentadas, reforça-se também a importância da realização de pesquisas cuidadosas, necessárias para melhorar a compreensão de como as potencialidades desta metodologia podem ser desenvolvidas, e como é possível alcançálas.

\section{PERCURSO METODOLÓGICO}

\subsection{A amostra e as hipóteses de investigação}

A investigação desenvolvida consistiu na realização de um estudo quasi-experimental com duas fases de recolha de dados (fase I e fase II). A fase I de recolha de dados ocorreu logo após o término da implementação do programa de intervenção e a fase II teve lugar três meses após o término da intervenção.

A amostra de conveniência, proveniente de duas turmas de alunos de Ciências Naturais, era constituída por 115 alunos a frequentar o 70 ano de escolaridade (3ํ ciclo do ensino básico português): 64 alunos pertencentes ao grupo experimental, com uma média de idade de 12.1 e 51 alunos que constituíam o grupo de controlo, com uma média de idade de 12.5. A maioria dos alunos da eram do sexo feminino: o grupo experimental constituído por 30 meninos e 34 meninas e o grupo de controlo composto por 21 meninos e 30 meninas.

Partindo da amostra de conveniência referida, foram definidos dois grupos, um grupo de controlo e um grupo experimental. A cada um dos grupos foi lecionada a temática "Fósseis e sua importância para a reconstituição da história da Terra" tendo-se aplicado duas metodologias de ensino diferentes (uma tradicional, baseada na leitura do manual escolar, no grupo de controlo; a ABP, com cenários problemáticos, no grupo experimental). 
Antes do início da intervenção, a ambos os grupos foi aplicado o pré-teste. Depois da Intervenção, a ambos os grupos, foram aplicados o pós-teste da fase I e o pós-teste da fase II. A aplicação de cada um dos testes teve a duração de 45 minutos. Os alunos foram alertados para o facto de o teste não ter qualquer influência na sua avaliação escolar, bem como para a necessidade e importância de pensarem e refletirem sobre cada resposta.

O estudo teve com base um conjunto de hipóteses de trabalho previamente formuladas. Essas hipóteses são as seguintes:

- H1: Os alunos sujeitos a uma metodologia de ABP têm significativamente melhores resultados no teste cognitivo realizado imediatamente após a intervenção (fase I) do que os alunos do grupo de controlo.

- H2: Os alunos sujeitos a uma metodologia de ABP têm significativamente melhores resultados no teste cognitivo realizado três meses após a intervenção (fase II) do que os alunos do grupo de controlo.

- H3: Os alunos sujeitos a uma metodologia de ABP apresentam uma maior e significativa retenção de conhecimento substantivo entre os diferentes momentos de aplicação do teste cognitivo (da fase de pré-teste para a fase I e da fase I para a fase II).

\subsection{O Programa de Intervenção}

O programa de intervenção foi desenvolvido de acordo com as indicações do currículo de Ciências Naturais português. Foi desenvolvido um cenário de problematização relacionado com fósseis e a reconstrução da história da Terra, inserido na temática "A Terra conta a sua História - Os fósseis e a sua importância para a reconstituição da História da Terra".

O cenário foi apresentado aos alunos do grupo experimental nas aulas de Ciências Naturais. No grupo de controlo a mesma temática foi lecionada através do ensino expositivo. Durante a implementação do cenário de problematização nas aulas de Ciências Naturais, promoveu-se também o questionamento e os alunos pesquisaram soluções para o problema, através da ajuda e mediação do professor. A etapa de investigação envolveu também a realização de um trabalho prático de modelação para compreensão do fenómeno de fossilização. Esta atividade prática foi levada a cabo pelos alunos, tendo-se potenciado o processo de aprendizagem, o desenvolvimento de competências de investigação e o raciocínio científico. 
Todo o processo de aprendizagem do conhecimento cognitivo enquadrado no currículo se centrou na compreensão e mobilização de conhecimentos que poderão ser aplicados a novas situaçõesproblema. Assim, depois da investigação os alunos foram desafiados a realizar uma outra atividade que exigia a aplicação do conhecimento e das competências que haviam sido desenvolvidas, permitindo, assim, a reconceptualização e a consolidação dos objetivos da aprendizagem.

As aulas relativas à lecionação da temática avaliada (Fósseis e sua importância para a reconstituição da história da Terra) ocorreram durante o primeiro trimestre do ano letivo 2012/2013. No grupo de controlo as aulas foram dadas de acordo com uma metodologia mais tradicional, enquanto no grupo experimental foi aplicado um cenário de problematização, enquadrado numa metodologia ABP. No total foram lecionadas três aulas de Ciências Naturais com a duração de 45 minutos cada uma. Foram fornecidas aos alunos as fontes de pesquisas necessárias para a realização das atividades propostas. Segundo as recomendações da ABP, os alunos trabalharam colaborativamente, distribuídos em grupos de quatro ou cinco elementos, e o seu trabalho foi mediado pelo professor, desempenhando a função de tutor.

O professor que dinamizou e lecionou as aulas aos participantes do grupo experimental estava familiarizado com a metodologia $\mathrm{ABP}$ e, tinha conhecimento do seu papel como tutor durante a aplicação do programa de intervenção.

\subsection{Testes cognitivos: validade e fidelidade}

Neste estudo foi aplicado um teste cognitivo em três momentos distinto e já referidos. Tendo em conta a temática selecionada para a aplicação do cenário “A Terra conta a sua História - Os fósseis e a sua importância para a reconstituição da História da Terra", e as recomendações do currículo de Ciências Naturais para o 7ํan ano do ensino básico, reuniu-se cuidadosamente um conjunto de questões que integraram o teste cognitivo. Estas questões incluíram perguntas de escolha múltipla, correspondências, ordenação de sequências, questões de resposta curta e de desenvolvimento de raciocínio. Todas as questões elaboradas pretendiam avaliar o conteúdo conceptual desenvolvido durante a implementação do programa de intervenção. $O$ teste foi validado por três docentes de Ciências Naturais, que aferiram, também, os critérios de correção. Os testes também foram corrigidos pelos mesmos três docentes. Após a correção individual por 
cada um dos avaliadores, este juntaram-se para discutirem alguns detalhes em que não estavam totalmente de acordo assegurando, assim, a fidelidade dos resultados.

\subsection{Estado inicial da amostra}

A situação inicial dos alunos da amostra foi averiguada antes da implementação da intervenção, através do cálculo das médias obtidas no pré-teste e da significância das diferenças desse valor entre o grupo de controlo e o grupo experimental. A análise foi feita recorrendo à versão 22 do programa estatístico SPSS. Foi realizado um teste estatístico não paramétrico cujo objetivo era analisar os resultados dos alunos $\mathrm{n}$ o pré-teste, esperando-se que fossem semelhantes em ambos os grupos. Os resultados estão apresentados na tabela 1.

Tabela 1. Resultados do pré-teste cognitivo.

\begin{tabular}{ccc}
\hline Pré-teste & Grupo experimental & Grupo de controlo \\
\hline \hline Média & 20.1 & 15.70 \\
Desvio Padrão & 9.59 & 9.88 \\
Mínimo & 0.00 & 0.00 \\
Máximo & 43.00 & 47.00 \\
\hline
\end{tabular}

Fonte: Dados da investigação.

Os valores obtidos em ambos os grupos, demonstraram alunos com baixo rendimento escolar em ambos os grupos. Não obstante, a média obtida no pré-teste cognitivo era mais elevada no grupo experimental (20.1 versus 15.7) com um desvio padrão mais elevado no grupo de controlo (9.88 versus 9.59). Foi aplicado o teste Mann-Whitney para verificar se existiam diferenças significativas nos resultados do teste aplicado, em cada um dos grupos em estudo. Este teste mostrou uma diferença estatisticamente significativa entre estes grupos antes da intervenção $(U=1171.5, p$ $<0.05)$, tendo o grupo experimental demonstrado melhores resultados a nível cognitivo.

Apesar dos resultados obtidos, isto é, de em termos de sucesso escolar os grupos serem significativamente diferentes, existiram várias razões que nos encorajaram a prosseguir com a investigação. É de realçar:

i) a dificuldade em conseguir professores e alunos que participem voluntariamente em estudos de caráter educacional;

ii) a possibilidade da emergência de resultados relevantes dos questionários a aplicar após a intervenção; 
iii) o facto da equipa de investigação ter assumido um compromisso ético de ajudar os professores a aprender e a aplicar a metodologia ABP.

\section{RESULTADOS}

Tendo em consideração as hipóteses formuladas no início do estudo, foi realizada uma análise estatística dos dados, suportada por teste não paramétricos. Os resultados obtidos são apresentados de seguida devidamente organizados em três subsecções. Alguns resultados parciais foram Estes já comunicados num congresso internacional (VASCONCELOS e TORRES, 2014).

\subsection{Comparação dos resultados dos alunos do pré-teste para o pós-teste (fase I)}

Como já foi referido, na fase I de recolha de dados, aplicou-se o pós-teste cognitivo imediatamente após o término da implementação do programa de intervenção. Após uma análise estatística dos dados recolhidos, obtiveram-se os resultados seguintes, organizados na tabela 2.

Tabela 2. Resultados do pós-teste cognitivo aplicado na fase I da intervenção.

\begin{tabular}{ccc}
\hline Pós-teste I & Grupo experimental & Grupo de controlo \\
\hline \hline Média & 41.2 & 33.30 \\
Desvio padrão & 19.11 & 15.69 \\
Mínimo & 15.00 & 8.00 \\
Máximo & 84.00 & 69.00 \\
\hline
\end{tabular}

De acordo com os dados da tabela 2, a média do pós-teste é mais elevada no grupo experimental, apesar de ambos os grupos continuarem a mostrar resultados baixos. Recorreu-se ao teste MannWhitney para verificar se existiam diferenças significativas nos resultados dos testes aplicados, em cada um dos grupos em estudo. Com a aplicação deste teste obteve-se uma diferença estatisticamente significativa nos dois grupos após a aplicação dos cenários geológicos $(U=$ $1271.500, \mathrm{p}<0.05)$. Estes resultados mostram que as melhorias verificadas a nível cognitivo depois da intervenção definem grupos com níveis de sucesso distintos, verificando-se que o grupo experimental atinge melhores resultados. 
O teste Wilcoxon foi usado para verificar se a diferença entre as médias do pré-teste e do pósteste da fase I, em cada grupo, eram estatisticamente diferentes. O grupo experimental demonstrou uma melhoria nas médias dos dois testes cognitivos, aumentando de 20.1 no préteste para 41.2 no pós-teste da fase I. A diferença obtida era estatisticamente significativa ( $Z$ =6.923, $\mathrm{p}<0.05)$. Para o grupo de controlo, a média melhorou também, de 15.7 no pré-teste cognitivo para foi 33.3 no pós-teste da fase I. A diferença obtida foi igualmente estatisticamente significativa $(Z=-6.166, p<0.05)$.

\subsection{Comparação dos resultados dos alunos no pré-teste e no pós-teste (fase II)}

Na fase II ocorreu nova aplicação de um pós-teste cognitivo três meses após o término da implementação do programa de intervenção. Os dados recolhidos foram sujeitos ao mesmo tipo de análise estatística, e na tabela 3 apresentamos valores da estatística descritiva desses resultados.

Tabela 3. Resultados do pós-teste cognitivo na fase II.

\begin{tabular}{ccc}
\hline Pós-teste II & Grupo experimental & Grupo de controlo \\
\hline \hline Média & 39.2 & 26.8 \\
Desvio padrão & 17.33 & 13.67 \\
Mínimo & 14.00 & 4.00 \\
Máximo & 76.00 & 61.00 \\
\hline
\end{tabular}

A média no pós-teste da fase Il é mais elevada no grupo experimental, apesar de ambos os grupos manterem os baixos resultados escolares. Aplicou-se o teste Mann-Whitney para verificar se existiam diferenças significativas nos resultados dos testes aplicados, nos grupos em estudo. Este teste demonstrou uma diferença estatisticamente significativa entre ambos os grupos após a aplicação dos cenários geológicos $(U=957.500, p<0.05)$. Desta forma, os resultados demonstram, mais uma vez, que as melhorias cognitivas três meses após o término da intervenção definem novamente grupos com níveis de sucesso distintos, revelando que o grupo experimental apresenta melhores resultados.

O teste Wilcoxon foi usado para verificar se as diferenças entre as médias do pré-teste e do pósteste da fase II eram estatisticamente significativas nos grupos em estudo. O grupo experimental mostrou uma melhoria na média dos testes cognitivos, verificando-se um aumento de 20.1 para 
39.2, e a diferença obtida era estatisticamente significativa $(Z=-6.762, p<0.05)$. Para o grupo de controlo, a média obtida no pré-teste cognitivo foi 15.7 e no pós-teste da fase II foi 26.8 . A diferença obtida foi também estatisticamente significativa $(Z=-5.423, p<0.05)$.

Tais resultados significam que três meses após a intervenção, a retenção do conhecimento substantiva é igualmente significativa nos dois grupos, embora o grupo experimental mostre valores significativamente mais elevados na resposta ao teste cognitivo.

\subsection{Comparação dos resultados dos alunos no pós-teste da fase l e no pós-teste da fase II}

Contudo se analisarmos a retenção do conhecimento do pós-teste da fase I (tabela 2) para o pósteste da fase II (tabela 3) verificamos que ambos os grupos descem o valor médio de resultados três meses após a intervenção. O grupo experimental desce de 41.2 para 39.2 e o grupo de controlo desce de 33.3 para 26.8 valores. Porém essa descida não é estatisticamente significativa para o grupo experimental $(Z=-1930.0, p<0.054)$. No entanto, no grupo de controlo essa diferença é estatisticamente significativa $(Z=-3.605, p<0.000)$. Tal resultado, demonstra que a retenção foi superior no grupo sujeito a ABP.

\section{DISCUSSÃO DOS RESULTADOS}

Após a análise estatística realizada aos resultados dos alunos nos testes aplicados nas duas fases de intervenção (fase I e fase II), e face aos valores obtidos nos testes não paramétricos realizados, algumas ilações podem ser retiradas. A primeira hipótese $[\mathrm{H} 1:$ Os alunos sujeitos a uma metodologia de ABP têm significativamente melhores resultados no teste cognitivo realizado imediatamente após a intervenção (fase I) do que os alunos do grupo de controlo] não é suportada pelos resultados.

Através dos dados obtidos verifica-se que os alunos melhoraram o seu desempenho do pré-teste para o pós-teste I, mas esta situação verifica-se tanto no grupo experimental como no grupo de controlo, sendo a melhoria em ambos estatisticamente significativa. A segunda hipótese [H2: Os alunos sujeitos a uma metodologia de ABP têm significativamente melhores resultados no teste cognitivo realizado três meses após a intervenção (fase II) do que os alunos do grupo de controlo] 
também não é suportada pelos resultados. Verifica-se mais uma vez, que apesar do desempenho dos alunos do grupo experimental ser melhor do que os alunos do grupo de controlo, em ambos os casos as melhorias nos testes são estatisticamente significativos. Desta forma é possível afirmar que a metodologia ABP permite a retenção de conhecimento a longo prazo, mas também isso acontece com uma metodologia mais expositiva. A terceira hipótese [H3: $\quad$ Os alunos sujeitos a uma metodologia de $A B P$ apresentam uma maior e significativa retenção de conhecimento substantivo entre os diferentes momentos de aplicação do teste cognitivo (da fase de pré-teste para a fase I e da fase I para a fase II)] é suportada pelos resultados do estudo.

A análise dos dados mostra que apesar de ambos os grupos terem piorado os seus desempenhos no pós-teste da fase II (facto que não surpreende, pois com o tempo a retenção da informação vai sempre diminuindo), essa diminuição é menor no grupo experimental, não sendo estatisticamente significativa. Pelo contrário, no grupo de controlo essa diminuição é estatisticamente significativa. Assim, os valores obtidos parecem indicar que os alunos do grupo experimental retêm mais conhecimentos substantivo aprendido com a intervenção da ABP do que os alunos do grupo de controlo. Pelo exposto, a metodologia ABP parece permitir uma retenção de conhecimento mais duradoura do que o ensino expositivo.

\section{CONSIDERAÇÕES FINAIS}

Tendo em consideração o objetivo principal deste estudo, que consistiu em analisar os benefícios de usar uma metodologia ABP, verificámos que os alunos de ambos os grupos, sujeitos a metodologias de ensino distintas, evidenciam melhorias a nível cognitivo. Além disso, os alunos sujeitos a uma metodologia ABP parecem evidenciar uma maior retenção de conhecimento.

Este trabalho mostrou que a introdução desta nova metodologia de ensino, no meio de um sistema educacional predominantemente tradicional, não causa nenhuma perturbação no desenvolvimento de conhecimento, como também foi verificado por Carrió e colaboradores (2011) e Antepohl e Herzig (1999). Note-se que esta preocupação é motivo de crítica, confusão e conflito entre os professores sempre que se quer implementar novas metodologias de ensino. Com este estudo foi possível demonstrar que essas preocupações são infundadas. Além disso, os 
alunos têm a oportunidade de realizar atividades de investigação e realizar tarefas que beneficiam o desenvolvimento do seu raciocínio científico. Essa oportunidade não oferecida pelo ensino expositivo, que apela apenas para a memorização mecânica e a reprodução retórica de conteúdos escolares.

Da mesma forma que Wong e Day (2008) constataram haver maior retenção do conhecimento em alunos sujeitos à metodologia $\mathrm{ABP}$, também os resultados por nós obtidos vão ao encontro deste pressuposto. Embora as classificações não tenham sido muito elevadas, os resultados nos testes cognitivos foram mais positivos no grupo experimental do que no grupo de controlo, sendo a diminuição do teste I para o II menor no grupo experimental. Portanto, relativamente à retenção do conhecimento os resultados demonstram que é maior no grupo experimental, no qual foi aplicada a metodologia ABP.

Este estudo revela que a implementação da metodologia ABP para além de promover o sucesso académico dos alunos auxilia os alunos numa maior retenção do conhecimento substantivo ao longo do tempo. Contudo, como é referido por Vasconcelos e colaboradores (2012), apesar de todas as vantagens metodológicas da $A B P$, os alunos estão ainda pouco familiarizados com o funcionamento e as particularidades de todo o processo. Este fator dificulta a dinâmica da sala de aula, exigindo assim, um reforço maior e mais frequente da ABP nas aulas de ciências, de modo a reafirmar o seu potencial educativo.

Os resultado obtidos levam-nos a referir que é fundamental o desenvolvimento de outros estudos de caráter longitudinal, de forma a corroborar a importância da ABP na promoção da retenção do conhecimento em períodos de tempo maiores do que as metodologias mais tradicionais.

\section{AGRADECIMENTOS}

Esta investigação foi realizada no âmbito do projeto "Educação em Ciência para a cidadania através da Aprendizagem Baseada na Resolução de Problemas" (PTDC/CPECED/108197/2008), financiado pela FCT no âmbito do Programa Operacional Temático Fatores de Competitividade (COMPETE) do Quadro Comunitário de Apoio III da União Europeia, e co-financiado pelo Fundo Comunitário Europeu (ERDF/ FEDER). 


\section{REFERÊNCIAS}

ABD-EL-KHALICK, F. et al. . Inquiry in Science Education: International Perspectives. Science Education, v. 88, n. 3, p. 397- 419, 2004.

ALLEN, D. E.; DONHAM, R. S.; BERNHARDT, S. A. Problem-based learning. New Directions for Teaching and Learning, v. 128, p. 21-29, 2011.

ALLEN, D. E.; DUCH, B. J.; GROH, S. E. The Power of Problem-Based Learning in Teaching Introductory Science Courses. In WILKERSON, L.; GIJSELAERS W. H. (Org.). Bringing Problem-Based Learning to Higher Education: Theory and Practice. New Directions for Teaching and Learning Series, San Francisco: JosseyBass, 1996.

ANTEPOHL, W.; HERZIG, S. Problem-Based Learning versus Lecture-Based Learning in a course of basic pharmacology: a controlled, randomized study. Medical Education, v. 33, p. $106-113,1999$.

BARREL, J. Problem-Based Learning: An inquiry approach. Thousand Oaks: Corwin Press, 2007.

CARRIÓ, M.; LARRAMONA, P.; BAÑOS, J.E.; PÉREZ. J. The effectiveness of the hybrid problem-based learning approach in the teaching of biology: a comparison with lecture-based learning. Journal of Biological Education, v. 45, n. 4, p. 229-235, 2011

DOLMANS, D. H. J. M.; DE GRAVE, W.; WOLFHAGEN, I. H. A. P.; VLEUTEN C. P. M. van der. Problem-based learning: future challenges for educational practice and research. Medical Education, v. 39 n. 7, p. 732-741, 2005.

GALLAGHER, S. A.; STEPIEN, W. J.; SHER, B. T.; WORKMAN, D. Implementing problem-based learning in science classrooms. School Science and Mathematics, v. 95, n. 3, p. 136- 146, 1995.

HMELO-SILVER, C. E. Problem-Based Leaning: What and How do Students Learn? Educational Psychology Review, v. 16, n. 3, p. 235-266, 2004.

KIM, M.; TAN, A. L.; TALAUE, F. T. New Vision and Chalenges in Inquiry-Based Learning Curriculum Change in Singapore. International Journal of Science Education, v. 35, n. 2, p. 289- 311, 2013.

MACKINNON, M. M. Core elements of student motivation in problem-based learning. New Directions for Teaching and Learning, v. 78, p. 49-58, 1999.

NATIONAL RESEARCH COUNCIL. Inquiry and the National Science Education Standards: A guide for teaching and learning. Washington: National Academic Press, 2008.

PARIS, S. G.; PARIS, A. H. Classroom applications of research on self-regulated learning. Educational Psychologist, v. 36, n. 2, p. $89-10,2001$.

PRINCE, K. J. A. H.; VAN EIJS, P. W. L. J.; BOSHUIZEN, H. P. A.; VAN DER VLEUTEN, C.P. M.; SCHERPBIER, A. J. J. A. General competencies of problem-based learning (PBL) and non-PBL graduates. Medical Education, $n$. 39, p. 394- 40, 2005.

SADEH, I.; ZION, M. The Development of Dynamic Inquiry Performances within an Open Inquiry Setting: A Comparison to Guided Inquiry Setting. Journal of Research in Science Teaching, v. 46, n. 10, p. 1137- 1160, 2009.

SAVIN-BADEN, M. Understanding the impact of assessment on students in Problem-Based Learning. Innovations in Education and Teaching International, v. 41, n. 2, p. 221 - 233, 2004.

TORRES, J.; PRETO, C.; VASCONCELOS, C. PBL Environmental Scenarios: An Analysis of Science Students and Teachers Questioning. Journal of Science Education, v. 14, n. 2, p. 71-74, 2013.

VASCONCELOS, C. Teaching Environmental Education through PBL: Evaluation of a Teaching Intervention Program. Research in Science Education, v. 42, n. 2, p. 219-232, 2012. 
VASCONCELOS, C.; ALMEIDA, A. Aprendizagem Baseada na Resolução de Problemas: Propostas de trabalho para Ciências Naturais, Biologia e Geologia. Porto: Porto Editora, 2012.

VASCONCELOS, C.; AMADOR, M. F.; SOARES, R. B.; PINTO, T. F. Questionar, investigar, e resolver problemas: reconstruindo cenários geológicos. Investigações em Ensino de Ciências, v. 17, n. 3, p. 709 - 720, 2012.

VASCONCELOS, C.; TORRES, J. Problem-Based and Lecture-Based Learning: a quasi-experimental study with natural sciences students. In ESERA 2013 Proceedings EBook, (in press) 2014, Nicosia: Cyprus, Proceedings.

WONG, K. K. H.; DAY, J. R. A Comparative Study of Problem-Based and Lecture-Based Learning in Junior Secondary School Science. Research in Science Education, v. 39, p. 625 - 642, 2008.

YOON, H.; WOO, A. J.; TREAGUST, D.; CHANDRASEGARAN, A. The Efficacy of Problem-based Learning in an Analytical Laboratory Course for Preservice Chemistry Teachers. International Journal of Science Education, v. 36, n. 1, p. 79- 102, 2014. 